Este estudio constituye la mayor base de datos sobre la prevalencia de VIH en trabajadoras sexuales en América Latina y complementa uno similar realizado en hombres que tienen sexo con hombres. Estos resultados demuestran el papel cada vez mayor que desempeña el consumo de drogas ilegales en la transmisión de esta infección en la población sexualmente activa. Se deben diseñar intervenciones locales efectivas enfocadas hacia la salud pública y la educación, que alcancen a este vulnerable segmento de la población. Esas intervenciones se deben dirigir no solo al control de la transmisión del VIH, sino también al de otras enfermedades de transmisión sexual y deben ajustarse a las particularidades de cada región y país, en especial a los patrones sexuales y conductuales predominantes y a los factores de riesgo específicos de cada localidad. (Bautista CT, Sánchez JL, Montano SM, Laguna-Torres A, Suárez L, et al. Seroprevalence of and risk factors for HIV-1 infection among female commercial sex workers in South America. Sex Transm Infect. 2006;82:311-6.)

\section{Los determinantes sociales de la salud, la posición socioeconómica y la inequidad en salud}

El surgimiento de modelos teóricos acerca de los determinantes sociales de la salud ha añadido más ambigüedad conceptual a la comprensión de la inequidad social en salud, ya que con frecuencia no es posible distinguir claramente entre la posición socioeconómica y esos determinantes. Ya sea que las inequidades sociales en salud se basen en las diferencias sanitarias o en las diferencias de los determinantes sociales de la salud que están asociadas con la posición socioeconómica, los responsables de tomar las decisiones políticas deben conocer la importancia que tiene para la salud la posición socioeconómica. La falta de claridad en los informes de los investigadores es una de las razones que impiden que sus investigaciones tengan un mayor efecto positivo sobre la elaboración de políticas sanitarias. Esto no solo se debe a la gran cantidad de modelos, sino a la imposibilidad de definir en muchos de ellos si la posición socioeconómica es un concepto teórico independiente o si está asociado con los determinantes sociales.

En este artículo se expone cómo esa ambigüedad en la interpretación de la posición socioeconómica y de los determinantes sociales de la salud, así como el solapamiento conceptual de ambos términos, contribuyen a la falta de la necesaria claridad en la información sobre la salud pública.

El empleo de un solo modelo teórico para explicar la salud y las inequidades sociales en la salud puede hacer más difícil distinguir entre los determinantes sociales de la salud y los procesos sociales que determinan la distribución social desigual de esos determinantes. Por otra parte, no está claro si las clases sociales constituyen una dimensión de la posición socioeconómica, a pesar de que muchos autores aceptan que son conceptos independientes. Las clases sociales no son una propiedad preestablecida de las personas, sino una relación social creada por las sociedades y como tal, preceden lógica y materialmente a su expresión en la distribución de los ingresos, la riqueza, la educación y el estatus social. Es precisamente para referirse a estos componentes de bienestar social que se utiliza el término "posición socioeconómica".

También es necesario ponerse de acuerdo sobre lo que se entiende por determinantes sociales de la salud y si estos determinantes constituyen un concepto diferente de la posición socioeconómica. La situación socioeconómica es un importante factor de predicción de la exposición a muchos riesgos considerados determinantes sociales de la salud. Algunos autores consideran que el término "social" debe reservarse para designar los procesos de distribución de los peligros materiales, las conductas saludables y los factores psicosociales en los diversos grupos socioeconómicos.

Por último, es necesario identificar las dimensiones e indicadores más apropiados de la posición socioeconómica para evaluar el efecto de las intervenciones dirigidas a reducir las inequidades. Si se acepta que cada dimensión de la posición socioeconómica puede influir sobre la salud por diferentes vías y de esa forma ser más o menos trascendente para la salud, ¿puede emplearse cualquiera de esas dimensiones para evaluar el resultado de una intervención dirigida a reducir la inequidad económica? ¿O las dimensiones de la posición socioeconómica que se deben emplear dependen del tipo de intervención? ¿Cuál o cuáles de las diversas dimensiones de la posición socioeconómica se deben utilizar? ¿Los ingresos, el nivel educacional o algún indicador de la clase social? Estas preguntas no tienen una respuesta sencilla, pero es necesario responderlas antes de poder evaluar el desempeño de una intervención.

En conclusión, para resolver las ambigüedades analizadas se propone satisfacer los siguientes requisitos básicos: alcanzar un consenso sobre las dimensiones que reflejan la posición socioeconómica; acordar qué se debe considerar como determinante social de la salud y si estos determinantes son un concepto teórico independiente de la posición socioeconómica; y finalmente, establecer cuáles dimensiones e indicadores de la posición socioeconómica son las más apropiadas para evaluar las intervenciones dirigidas a reducir las inequidades 
en salud. (Regidor E. Social determinants of health: a veil that hides socioeconomic position and its relation with health. J Epidemiol Community Health. 2006;60:896-901.)

\section{Efecto independiente de la exposición pasiva prenatal y posnatal al tabaco}

La exposición pasiva al tabaco (EPT), tanto prenatal como posnatal, está asociada con las afecciones respiratorias y el asma durante la infancia. Sin embargo, se desconoce la contribución individual de cada una de estas formas de EPT a los trastornos pulmonares en la población infantil. En este artículo se analiza el efecto que tiene sobre la función respiratoria la EPT prenatal y posnatal de niños en edad escolar de varios países con características geográficas, sociales y culturales muy diferentes entre sí.

Se utilizaron los datos de volumen y flujo respiratorios de ocho estudios transversales que forman parte del estudio Polución y Juventud (PATY), que abarcó 57363 niños de 10 países europeos, Rusia y América del Norte. En total se analizaron 22712 niños, de los cuales se contaba con la información sobre la asociación independiente entre los indicadores de la función pulmonar y la EPT en la etapa prenatal (hábito de fumar de la madre durante el embarazo) y posnatal (durante los primeros 2 años de vida del niño y en el momento de la investigación). La información sobre la EPT se obtuvo mediante cuestionarios y la prueba de espirometría se realizó de manera estandarizada según el protocolo establecido por la Sociedad Estadounidense del Tórax y la Sociedad Europea de Enfermedades Respiratorias.

La frecuencia del hábito de fumar durante el embarazo varió entre 4,9\% en Alemania y 28,1\% en Holanda. Según la regresión logística se observó un aumento en el riesgo de disminución de la función pulmonar (31\% del flujo pico espiratorio y $40 \%$ del flujo medio espiratorio máximo) en los niños expuestos al tabaquismo de la madre durante el embarazo. En el modelo parcialmente ajustado, el efecto fue menor (15\% y 30\%, respectivamente), pero mantuvo su significación estadística.
A pesar de las diferencias geográficas, sociales y ambientales, se observó una gran coherencia entre los resultados de todos los estudios analizados. El hábito de fumar durante el embarazo tuvo un mayor efecto sobre los parámetros de flujo que en el volumen espirado durante el primer segundo de una espiración forzada.

El efecto de la EPT encontrado en este estudio afectó poco a la función pulmonar media de la población, pero contribuyó a aumentar el porcentaje de niños con actividad pulmonar deficiente. El efecto del hábito de fumar durante el embarazo y de la EPT actual se redujo después de ajustar por algunos posibles factores de confusión. Sin embargo, algunos de esos factores, como el hacinamiento o el bajo estatus socioeconómico (medido según el nivel educacional de los padres), pueden haber influido en la magnitud del efecto de la EPT por el efecto añadido de las infecciones respiratorias que pudieran haber sido favorecidas por la EPT.

Se encontró un mayor efecto negativo, aunque pequeño, de la EPT en niños que en niñas. El hábito de fumar afectó más a los niños que no padecían de asma que a los asmáticos, aunque esto pudo deberse al subdiagnóstico del asma en los niños de familias de menor estatus socioeconómico, en las que la EPT tiende a ser mayor. No se observó una clara tendencia relacionada con la edad, lo que resulta interesante, ya que esto hubiera reflejado el debilitamiento del efecto sufrido por haber estado sometido a EPT solamente durante el embarazo.

En conclusión, los resultados de este estudio confirman en una gran muestra multinacional el efecto negativo que provoca el hábito de fumar de las mujeres durante el embarazo sobre la actividad pulmonar posterior de sus hijos. Este efecto fue mayor que el daño causado por la EPT durante la infancia. Además, demuestran que ambos efectos son independientes. Tomando en cuenta el elevado número de niños expuestos, estos resultados indican que la EPT, tanto durante el embarazo como en la infancia, constituye un grave problema de salud pública. (Moshammer H, Hoek G, LuttmannGibson H, Neuberger MA, Antova T, Gehring U, et al. Parental smoking and lung function in children. An international study. Tob Control. 2006;15: 294-301.) 